\title{
TGM3, a candidate tumor suppressor gene, contributes to human head and neck cancer
}

\author{
Xiangbing $\mathrm{Wu}^{1,2}$, Wei Cao ${ }^{1,2}$, Xu Wang ${ }^{1,2}$, Jianjun Zhang ${ }^{1,2}$, Zhongjing $\mathrm{Lv}^{1,2}$, Xing Qin ${ }^{1,2}$, Yadi Wu ${ }^{2}$ \\ and Wantao Chen ${ }^{1,2^{*}}$
}

\begin{abstract}
Background: In our previous study using oligonucleotide microarrays, we revealed that transglutaminase 3 (TGM3) was remarkably down-regulated in head and neck cancer (HNC). However, the potential of TGM3 as a useful biomarker or molecular target for HNC is unclear.

Methods: The transcriptional and post-translational status of TGM3 in HNC cell lines and specimens was detected using real-time PCR and western blot analysis. Bisulfate-treated DNA sequencing was used to analyze the molecular mechanism of TGM3 gene silencing. In addition, the effects of TGM3 on the proliferation, colony formation and induction of apoptosis in vitro and tumorigenicity in vivo were investigated through exogenous expression of TGM3 in HNC cells. Immunohistochemistry was used to evaluate TGM3 expression in large HNC samples.

Results: TGM3 was down-regulated in HNC samples and cell lines $(P<0.0001)$. The hypermethylation of a promoter $\mathrm{CpG}$ island was one of the mechanisms of silencing the TGM3 gene in HNC. Exogenous expression of TGM3 in HNC cells could inhibit the proliferation and enhance the apoptosis of HNC cells in vitro and suppress tumor growth in vivo. In addition, TGM3 protein levels were strongly associated with the pathological differentiation of HNC tissues $(P=0.0037)$. Survival analysis revealed that low TGM3 expression was associated with worse overall survival $(P=0.0002)$, and TGM3 expression level was an independent predictor in patients with HNC.
\end{abstract}

Conclusions: The studies prove that TGM3, as a candidate tumor suppressor, contributes to the carcinogenesis and development of HNC and may serve as a useful biomarker for patients with HNC.

Keywords: Transglutaminase 3, Head and neck cancer, Tumor suppressor, Methylation, Prognostic predictor

\section{Introduction}

Head and neck cancer (HNC), squamous cell carcinoma accounts for over $90 \%$, is the sixth most common cancer in the world [1]. Despite continuous improvement in traditional treatments (surgery, radiotherapy and chemotherapy), the 5-year survival rate for patients with these devastating diseases has been unsatisfactory in the past three decades [2]. In an attempt to improve the outcomes of HNC, the application of novel and effective biomarkers for diagnosing and predicting HNCs is so important and urgent. Recently, certain molecular biomarkers have been found to have promising diagnostic

\footnotetext{
* Correspondence: chenwantao2002@hotmail.com

'Department of Oral and Maxillofacial-Head and Neck Oncology and Facuty of Oral and Maxillofacial Surgery, Ninth People's Hospital, Shanghai Jiao Tong University School of Medicine, Shanghai 200011, China

${ }^{2}$ Shanghai Research Institute of Stomatology and Shanghai Key Laboratory of Stomatology, Shanghai 200011, China
}

and predicting potentials and are already used in oncological practice [3-6], whereas others necessitate further studies. As we have known, the development of $\mathrm{HNC}$ is a multistep carcinogenic processes that includes activation of several oncogenes and inactivation of tumor suppressor genes [7]. High-throughput microarray technology might be an efficient way to uncover clues to these processes and find biomarkers for the diagnosis, therapy and prognosis of HNC [8-10]. In our previous study [11], oligonucleotide microarrays (Affymetrix HG-U95Av2) were used to select differentially expressed genes between 22 pairs of head and neck squamous cell carcinoma (HNSCC) and normal epithelial tissues from the same donors. Remarkably, transglutaminase 3 (TGM3) was significantly down-regulated in HNSCCs.

TGM3, encoded by the TGM3 gene, is widely expressed in the small intestine, brain, skin and mucosa [12]. In the 
skin and mucosa, TGM3 is predominantly expressed in the suprabasal layers of the stratified squamous epithelium $[13,14]$. It has been demonstrated that TGM3 is essential for epidermal terminal differentiation and formation of the cornified cell envelope through cross-linking structural proteins such as involucrin, loricrin and small proline-rich proteins $[15,16]$. Recent studies have revealed that the down-regulation of the TGM3 gene is closely linked with a variety of human cancer types, including laryngeal carcinoma, esophageal and oral squamous cell carcinoma (OSCC) [17-19]. Moreover, Uemura et al. reported that TGM3 was identified as a novel prognostic indicator in ESCC and the prognostic performance of TGM3 was confirmed by immunohistochemistry in 76 ESCC cases [18]. In addition, Mendez et al. reported that the TGM3 gene was differentially expressed in nodepositive and node-negative primary tumors in patients with OSCC, implying that the decreasing TGM3 expression might contribute to the metastatic potential of OSCC [20]. However, the biological function and molecular mechanism of the TGM3 gene in cancer initiation and progression have not been reported. In addition, whether the TGM3 gene might be a valuable diagnostic or therapeutic biomarker for cancer, especially for $\mathrm{HNC}$, needs to be further investigated.

In our current study, we confirmed that the transcriptional and post-translational levels of TGM3 were downregulated in HNSCC cell lines and specimens compared with normal primary head and neck epithelial cells and paired adjacent normal tissues, by means of the realtime RT-PCR, semi-quantitative RT-PCR and western blotting. We further found that the hypermethylation of a promoter $\mathrm{CpG}$ island was one of the mechanisms contributing to the silencing of the TGM3 gene in HNSCC. Furthermore, we evaluated the effect of ectopic TGM3 expression on WSU-HN4, HN13 and HN30 cells, which are HNSCC-derived cell lines that lack endogenous TGM3 expression. We first provide evidence that the exogenous expression of TGM3 in HNSCC cell lines inhibits the proliferation, colony formation and induces the apoptosis in cancer cells in vitro and tumorigenicity in vivo. In addition, we demonstrate that low TGM3 expression is notably associated with poorly differentiated tumors and worse overall survival. These results suggest that TGM3 might be a candidate tumor suppressor contributing to $\mathrm{HNC}$ and could act as a valuable prognostic predictor for patients with $\mathrm{HNC}$.

\section{Results}

TGM3 is down-regulated in HNSCC samples and cell lines To confirm our high-throughput microarray data, semiquantitative RT-PCR and real-time RT-PCR analyses were performed to investigate the mRNA levels of TGM3 in 53 paired HNSCC specimens, 9 HNSCC cell lines, and normal primary head and neck epithelial cells. The TGM3 mRNA levels in the HNSCC cell lines were significantly lower than those of normal epithelial cells (Figure 1A and B). Meanwhile, TGM3 transcription levels were remarkably down-regulated in HNSCC tissues compared with the levels of paired adjacent normal tissues $(P<0.0001)$ (Figure 1D and E). Correspondingly, the protein levels of TGM3 were remarkably downregulated in 9 HNSCC cell lines and 7 HNSCC tissues compared with the levels in normal epithelial cells and the paired adjacent normal tissues using western blot analysis (Figure $1 \mathrm{C}$ and $\mathrm{F}$ ).

\section{The transcription of TGM3 is regulated by DNA promoter methylation in HNSCC samples and cell lines}

To investigate the molecular mechanism of TGM3 gene silencing in HNSCC tumor samples and cell lines, all of the above-mentioned HNSCC cell lines lacking TGM3 expression were treated with the demethylation agent 5-Aza-dC and the histone deacetylase inhibitor TSA. Real-time PCR analysis revealed a dose-dependent up-regulation of TGM3 mRNA expression after treatment with 5-Aza-dC (Figure 2A). However, there was no effect on the reactivation of TGM3 expression after treatment with TSA, indicating that histone deacetylation does not contribute to the transcriptional silencing of TGM3 in HNSCC cell lines (data not shown). To further confirm that the TGM3 gene was regulated through DNA hypermethylation, a putative CpG island, consisting of a $113 \mathrm{bp}$ fragment containing $9 \mathrm{CpG}$ sites located -6627 to -6515 bp relative to the transcriptional start site (TSS) was found by the MethPrimer online software (Figure 2B). Next, bisulfate-treated DNA sequencing was performed to examine the DNA methylation status of this $\mathrm{CpG}$ islands in 5 randomly selected HNSCC cell lines and 5 pairs of HNSCC tissues from 53 cases. The results showed that this $\mathrm{CpG}$ island was hypermethylated in the HNSCC cell lines relative to normal primary head and neck epithelial cells. Moreover, the methylation level of the $\mathrm{CpG}$ island was significantly higher in all $5 \mathrm{HNSCC}$ samples than in the paired adjacent normal tissues $(P<0.05$, Figure $2 C)$.

\section{Correlation between TGM3 protein level and clinicopathologic parameters and clinical outcome in patients with HNSCCs}

Using immunohistochemical staining to detect TGM3 expression levels, we observed that, in normal epithelial tissue, cytoplasmic and nuclear staining of TGM3 was expressed in keratinized layer, stratum granulosum and in a portion of stratum spinosum nearby stratum granulosum. Whereas weak or no staining of TGM3 was observed in basal layer and in a portion of stratum spinosum nearby basal layer. In HNSCC specimens, cytoplasmic and 

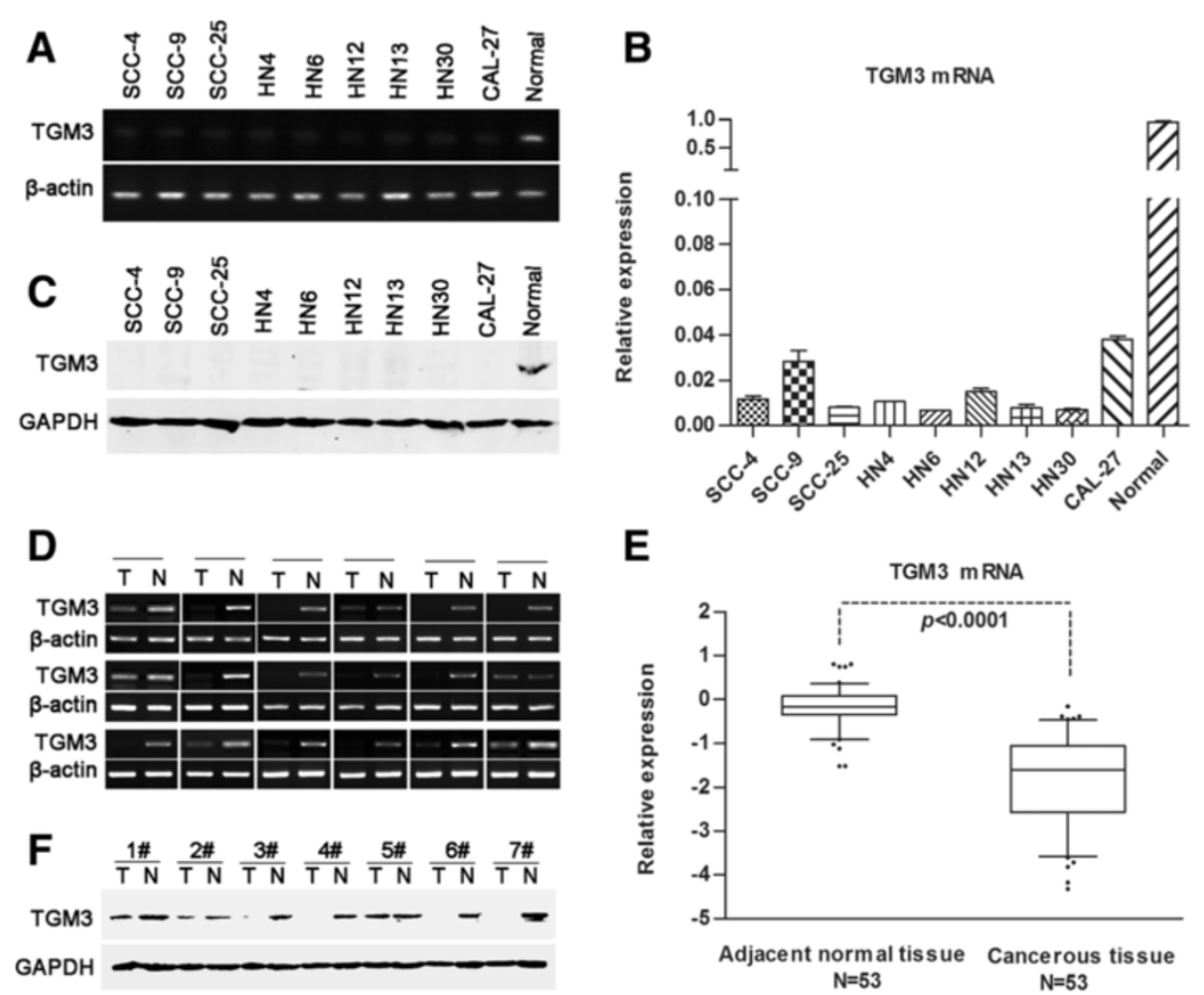

Figure 1 TGM3 mRNA and protein levels are illustrated in HNSCC specimens and cell lines. (A, B, C) TGM3 transcriptional and translational status in 9 representative HNSCC cell lines and primary normal epithelial cells (titled normal) was determined using semi-quantitative RT-PCR, real-time PCR and western blotting analysis. (D) Representative results of TGM3 mRNA levels from 18 pairs of HNSCC and their adjacent normal tissue. (E, F) TGM3 mRNA and protein levels were determined in 53 paired samples from patients with HNSCC and in 7 paired HNSCC samples using real-time PCR and western blot analyses ( $N$, adjacent normal tissue; $T$, tumor tissue).

nuclear staining of TGM3 was detected in well differentiated tumors, whereas weak or no staining of TGM3 was observed in moderately differentiated and poorly differentiated tumors (Figure 3A). There was positive association between TGM3 expression level and the pathological differentiation of the tumors $(P=0.0037)$, whereas no significant associations were determined between TGM3 expression pattern and gender $(P=0.9873)$, age $(P=0.0718)$, alcohol history $(P=0.1543)$, smoking history $(P=0.9860)$, TNM classification $(P=0.1015)$, lymph node metastasis $(P=0.2404)$, or anatomic site $(P=0.6781$, Figure $3 \mathrm{~B})$. Overall survival probability was estimated using KaplanMeier survival analysis to determine the correlation between TGM3 expression and clinical outcome in patients with HNSCCs. We observed that patients whose primary tumors expressed a low level of TGM3 had significantly poorer overall survival $(P=0.0002$, Figure $3 C)$. In univariate and multivariate Cox proportional analyses (Table 1), TGM3 expression status (hazard ratio, 0.286; 95\% CI, 0.103-0.796; $P=0.016$ ), together with pathological differentiation of the tumor (hazard ratio, 1.737; 95\% CI, $1.042-2.895 ; P=0.034$ ), was identified as an independent predictor of clinical outcome in patients with HNSCCs.
TGM3 Inhibits cell proliferation and colony formation To analyze the function of the TGM3 protein in HNSCC cells, we transiently transfected mammalian expression vectors containing TGM3 into the WSU-HN4, HN13 and HN30 cell lines, all of which lack endogenous TGM3 expression (Figure 4A). Our results showed that exogenous TGM3 expression notably suppressed the proliferation and colony-formation abilities of these cells relative to mock-transfected controls (Figure 4B and C). To further evaluate whether cell cycle distribution was altered, flow cytometry was performed in cells expressing exogenous TGM3. Nevertheless, there was no difference in cell cycle distribution between the cells transiently transfected with the pcDNA3.0-TGM3 vector and the pcDNA3.0 empty vector (data not shown).

\section{Induction of apoptosis in HNSCC cells by ectopic TGM3 expression}

To analyze whether the suppressive effect of the TGM3 protein on cells was caused by apoptosis, the fraction of TGM3-transfected WSU-HN4, HN13 and HN30 cells with positive staining for propidium iodide (PI) and Annexin $\mathrm{V}$ were assessed by flow cytometry. The fractions 


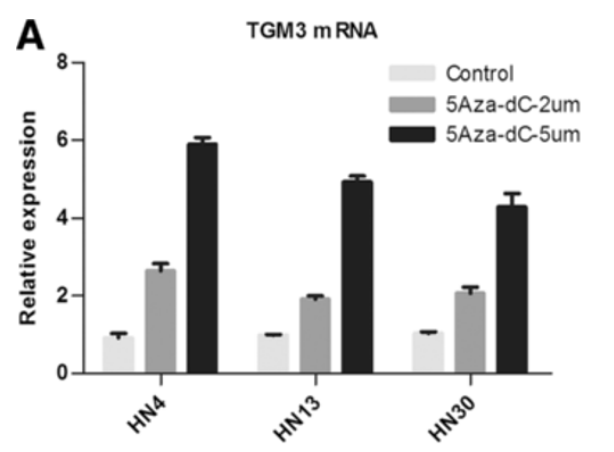

B

C
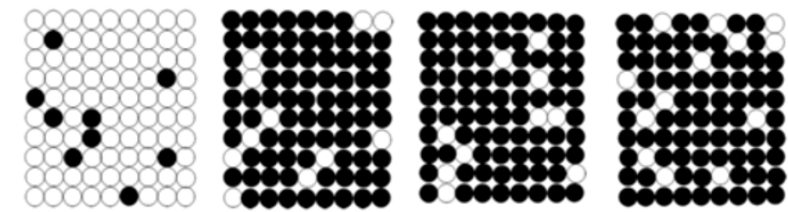

HN13

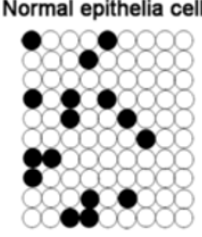

$10 \mathrm{~N}$

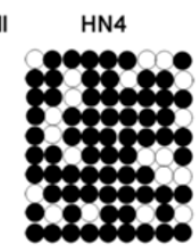

10T

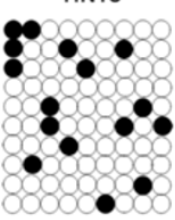

$11 \mathrm{~N}$

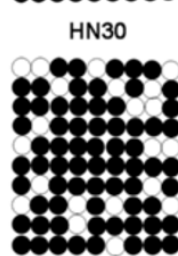

11T

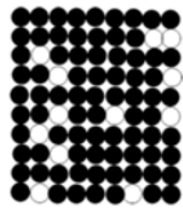

CAl-27

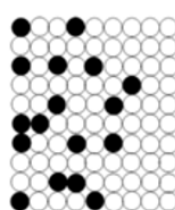

$12 \mathrm{~N}$

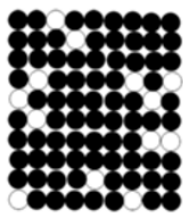

SCC-9

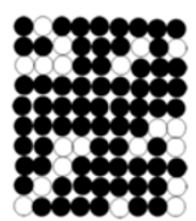

12T

Figure 2 Promoter region hypermethylation mediated the silencing of TGM3 in HNSCC. (A) Restoration of TGM3 expression by 5-Aza-dC. Three representative HNSCC cell lines were treated with $2 \mu \mathrm{M}$ or $5 \mu \mathrm{M}$ 5-Aza-dC for 5 days, and TGM3 transcriptional levels were determined by real-time RT-PCR. (B) Schematic representations of the location of the CpG islands in the upstream region of the TGM3 gene and the location of bisulfate-based sequencing within the $\mathrm{CpG}$ island. (C) Bisulfate-treated genomic DNA sequencing to detect the methylation status of the CpG islands in 5 HNSCC cell lines and 3 representative HNSCC samples compared with the methylation status in normal primary head and neck epithelial cells and paired adjacent normal tissues. White and black circles represent unmethylated and methylated CpGs, respectively.

of apoptotic cells (lower right (LR) fraction) in TGM3transfected WSU-HN4, HN13 and HN30 cells were $65.69 \%, 37.89 \%$ and $36.97 \%$, respectively, which were notably higher than those of the mock-transfected control cells (23.08\%, 15.57\%, 15.79\%) $(P<0.05$, Figure 5A). Furthermore, we also analyzed differences in specific apoptosisrelated proteins between cells transiently transfected with the pcDNA3.0-TGM3 vector and the pcDNA3.0 empty vector. As shown in Figure 5B, the protein levels of cleaved PARP and Bax were increased and the levels of full-length PARP, procaspase- 3 , procaspase- 8 and $\mathrm{Bcl}-2$ were reduced in TGM3-transfected HN4, HN13 and HN30 cells, compared to mock-transfected control cells, as detected by western blotting analysis.

\section{Ectopic expression of TGM3 suppresses tumor growth in vivo}

After evaluating the anti-tumor activity of TGM3 in vitro, we next analyzed whether exogenous TGM3 expression affected tumorigenicity in vivo using an HN30 xenograft model in BALB/C nude mice. One week after subcutaneously injecting HN30 cells, AdCMV-TGM3 and AdCMV-GFP was administered locally by multiple- center intratumor injection. As shown in Figure 6, the average tumor sizes with AdCMV-TGM3 treatment were significantly smaller than the control group $(P<0.0001)$.

\section{Discussion}

The limitations of conventional treatments in advanced, recurrent or distant metastases HNC have necessitated a search for novel potential approaches. Recently, several research groups have reported certain genes and signaling molecules that were potentially involved in HNC initiation and progression [21-24]. These molecular targets could provide significant clues for early diagnosis, prognosis and new targeted therapies [25-30]. In our previous study [11], we found that TGM3 was remarkably down-regulated in HNSCC compared with adjacent normal tissues by means of an oligonucleotide microarray analysis. In this study, we have first verified the microarray data by detecting the mRNA and protein levels of TGM3 in 9 HNSCC cell lines and 53 HNSCC specimens. Our findings were consistent with other reports, in which TGM3 was also found to be down-regulation in laryngeal carcinoma, esophageal SCC and OSCC [31-40]. Furthermore, we evaluated the clinical significance of TGM3 by 


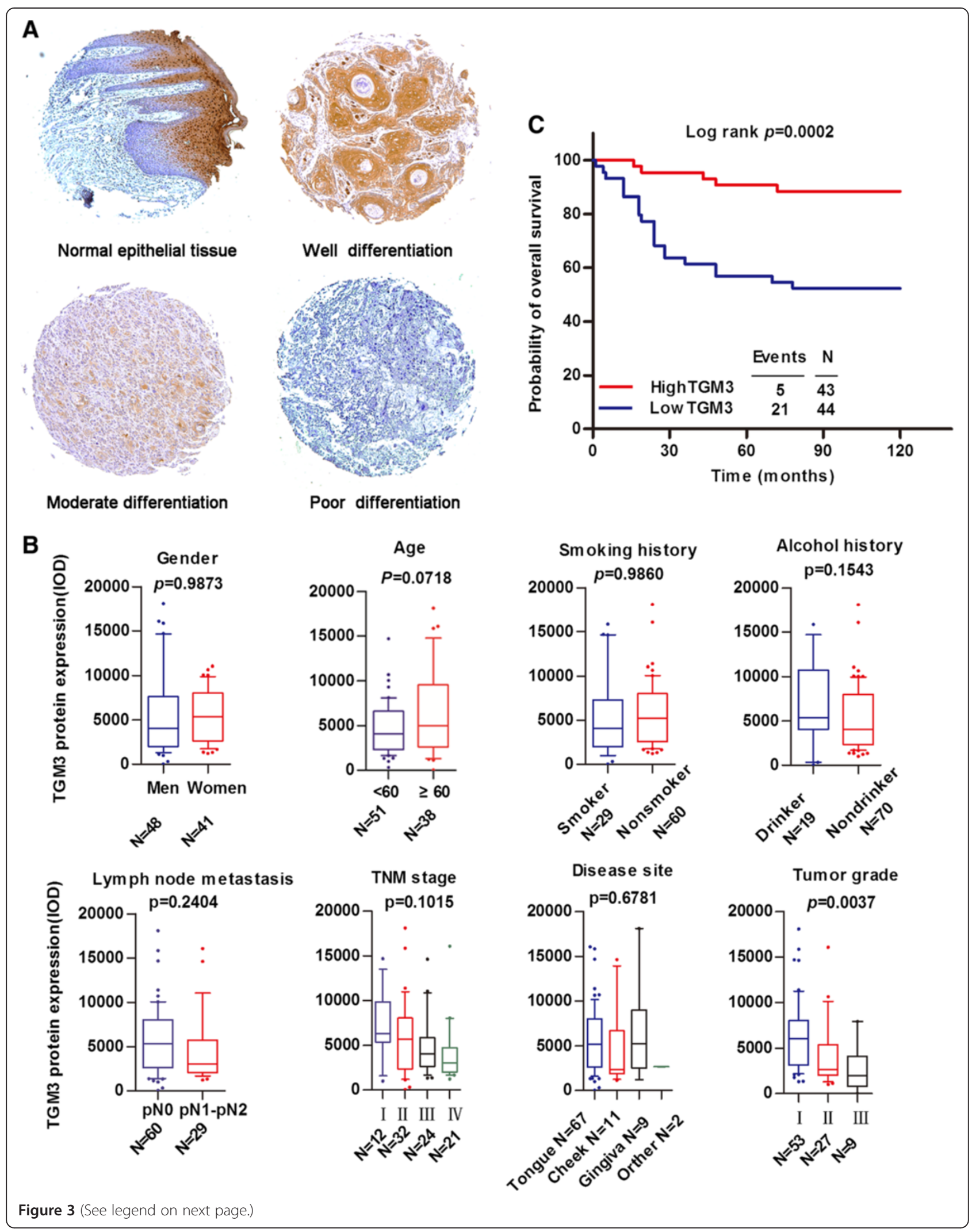


(See figure on previous page.)

Figure 3 Correlation between TGM3 expression and clinicopathological parameters and clinical outcome in patients with HNSCCs. (A) Representative images show immunohistochemical staining for TGM3 expression in normal epithelial tissue, well differentiated HNSCC, moderately differentiated HNSCC and poorly differentiated HNSCC. (B) The associations between TGM3 expression and clinicopathological factors were investigated in patients with HNSCCS (Twelve patients were excluded due to the samples shedding in the process of antigen retrieval and due to an insufficient number of tumor cells in the sections). (C) Kaplan-Meier survival curves illustrate the overall survival of patients with HNSCC relative to TGM3 expression (Two patients were excluded from survival analysis because of missing follow-up data).

analyzing the correlation between TGM3 expression pattern and clinicopathologic parameters and clinical outcome in patients with HNSCC. Our data demonstrated that the reduced expression of TGM3 in HNSCC was associated with poorer pathological tumor differentiation $(P=0.0037)$, consistent with the findings described in ESCC and OSCC $[19,38]$. Moreover, we observed that patients whose tumors expressed a low level of TGM3 had worse overall survival $(P=0.0002)$ and that TGM3 expression, by univariate and multivariate analyses, was an independent prognostic factor in patients with HNSCC. This result was consistent with the findings of Uemura, who reported that the 5-year disease-specific survival rate was $64.5 \%$ and $32.1 \%$, respectively, for patients with TGM3positive and TGM3-negative ESCC, and TGM3 was identified as independent predictor in patients with ESCC [18]. Although Mendez et al. reported that TGM3 expression was inversely correlated with lymph node metastasis of OSCCs [20], we did not observe a significant correlation

Table 1 Univariate and multivariate cox proportional hazards regression models for estimating overall survival

\begin{tabular}{|c|c|c|c|}
\hline Characteristics & HR & $95 \% \mathrm{Cl}$ & $P$ \\
\hline \multicolumn{4}{|l|}{ Univariate analysis } \\
\hline \multicolumn{4}{|l|}{ Overall survival } \\
\hline Age (<60 y vs $\geq 60$ y) & 0.633 & 0.282 to 1.419 & 0.267 \\
\hline Gender (male vs female) & 1.713 & 0.763 to 3.844 & 0.192 \\
\hline Smoking history (smoker vs nonsmoker) & 0.643 & 0.295 to 1.401 & 0.267 \\
\hline Alcohol history (drinker vs nondrinker) & 0.718 & 0.302 to 1.709 & 0.454 \\
\hline Tumor grade & 2.096 & 1.283 to 3.425 & 0.003 \\
\hline TNM stage & 1.784 & 1.181 to 2.696 & 0.006 \\
\hline $\begin{array}{l}\text { Lymph node metastasis } \\
\text { (pNO vs pN1 to pN2) }\end{array}$ & 2.514 & 1.153 to 5.481 & 0.020 \\
\hline TGM3 expression (high vs low) & 0.191 & 0.072 to 0.506 & 0.001 \\
\hline Disease site & 1.023 & 0.637 to 1.660 & 0.926 \\
\hline \multicolumn{4}{|l|}{ Multivariate analysis } \\
\hline \multicolumn{4}{|l|}{ Overall survival } \\
\hline Tumor grade & 1.737 & 1.042 to 2.895 & 0.034 \\
\hline TNM stage & 1.325 & 0.806 to 2.177 & 0.267 \\
\hline $\begin{array}{l}\text { Lymph node metastasis } \\
\text { (pNO vs pN1 to pN2) }\end{array}$ & 1.515 & 0.586 to 3.909 & 0.391 \\
\hline TGM3 expression (high vs low) & 0.286 & 0.103 to 0.796 & 0.016 \\
\hline
\end{tabular}

Abbreviations: $\mathrm{Cl}$ confidence interval; $H R$ hazard ratio; $p N$ pathological lymph node status; TNM tumor-lymph node-metastasis classification. between TGM3 expression and lymph node metastasis in our study. This inconformity is most likely due to the different type and number of tumor samples included in the different studies. In Mendez's research, the number of OSCC specimens was 11 and the type of samples selectively included 6 metastatic lymph node samples. In our study, the tumor specimens were 101 cases which were all primary HNSCC samples and the exclusion criteria of these samples included preoperative radiotherapy and/or chemotherapy, distant metastasis and recurrent tumors.

Although TGM3 down-regulation has been found in many cases, the molecular mechanism that causes the silencing of TGM3 expression in most cancers remains unclear. He et al. reported that the loss of heterozygosity within and near the TGM3 gene might lead to downregulation of TGM3 in laryngeal carcinoma [17]. Aside from genetic changes, epigenetic alterations including DNA methylation, histone modification and non-coding RNA regulation could account for the down-regulation of TGM3. To evaluate the regulation of the DNA methylation and histone deacetylation modifications on TGM3 expression in HNSCC, we treated HNSCC cell lines with the demethylation agent 5-Aza-dC and the histone deacetylase inhibitor TSA. Our results, consistent with Negishi's findings [19], showed a dose-dependent up-regulation of TGM3 mRNA expression after treatment with 5-Aza-dC and no change in TGM3 expression after treatment with TSA. Although TGM3 gene hypermethylation in OSCC cell lines was first found by Negishi et al. [19], a more detailed characterization of the DNA hypermethylation status of the TGM3 gene promoter in paired HNSCC samples and adjacent normal tissues has never been reported. Bioinformatics tools were utilized to search for putative CpG islands in the regulatory region of the TGM3 gene from $8,000 \mathrm{bp}$ upstream to $500 \mathrm{bp}$ downstream of the transcriptional start site. We have first identified a CpG island located at -6627 to -6515 bp (including 9 CpG sites) relative to the transcriptional start site. Furthermore, bisulfate-treated DNA sequencing revealed that the CpG island was hypermethylated in 5 HNSCC cell lines relative to normal primary head and neck epithelial cells, and the methylation level of this CpG island was significantly higher in 5 randomly selected HNSCC samples than in paired adjacent normal tissues. Based on our results and the findings reported by others [19], we propose that DNA hypermethylation in the 

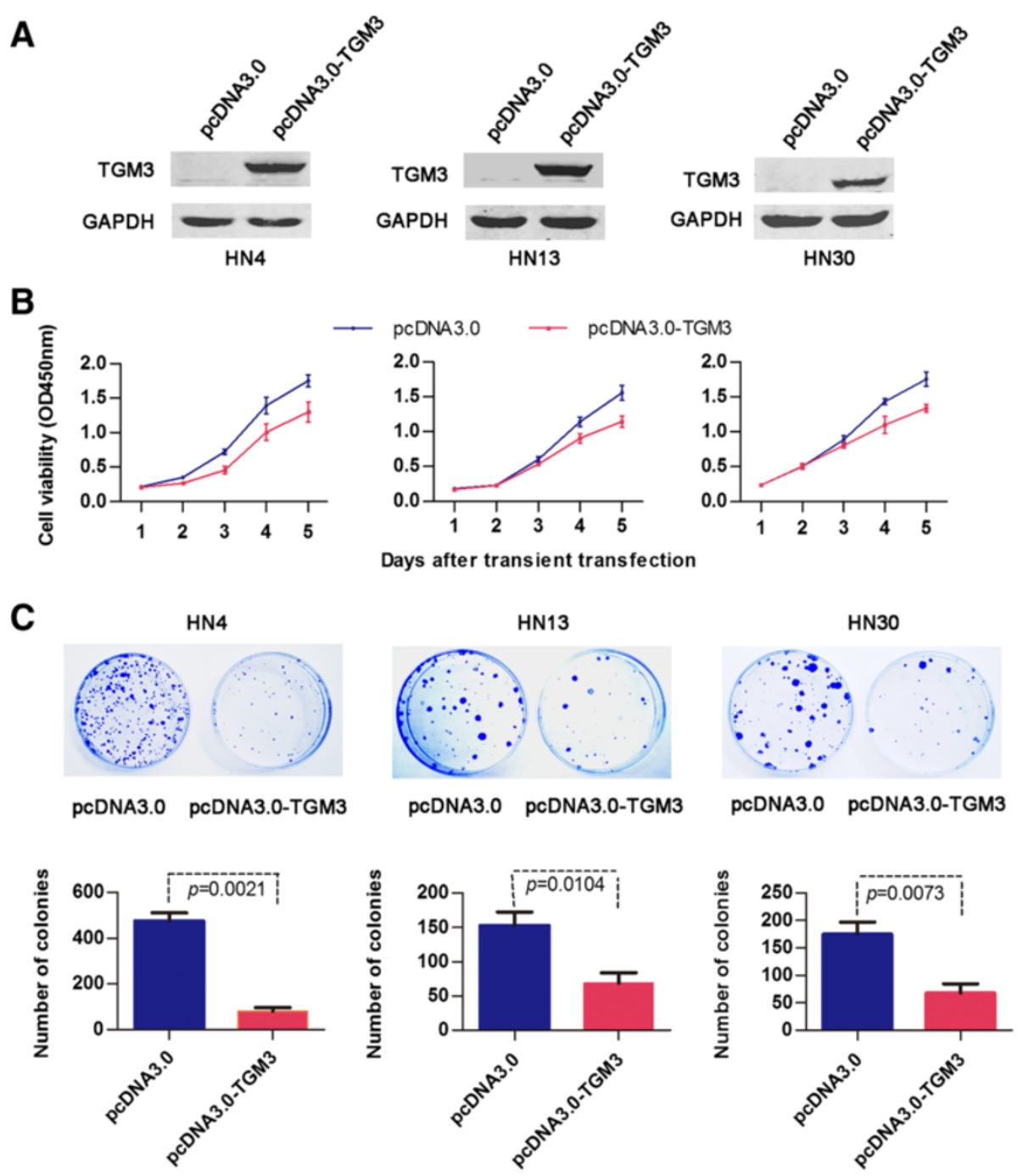

Figure 4 The effect of TGM3 on cell growth and colony formation of HNSCC cell lines. (A) Western blot analysis confirmed the exogenous expression of TGM3 in HN4, HN13 and HN30 cells. (B) The growth of HN4, HN13 and HN30 cells transfected with the pcDNA3.0 and pcDNA3.0-TGM3 vectors were analyzed using the CCK-8 kit; the symbols represent the mean values of triplicate tests (mean \pm SD). (C) Representative dishes show the inhibitory effect of TGM3 on colony formation compared with the empty vector control. All experiments were repeated at least 3 times.

TGM3 regulatory region contributes, at least partially, to the down-regulation of TGM3 in HNSCC.

Transglutaminases (TGMs) are a family of calciumdependent enzymes that catalyze the formation of isopeptide bonds $[41,42]$. TGM3 is epidermal transglutaminase, which is expressed predominantly in the suprabasal layers of the stratified squamous epithelium [43]. Although, the biological function of TGM3 in stratified squamous epithelium is very clear, in vitro and in vivo functional experiments of TGM3 on epithelial cancer cells have never been reported. In this study, we evaluated the functional role of the TGM3 gene in 3 HNSCC cell lines. Our results, for the first time, demonstrated that exogenous TGM3 expression could exert strong anti-proliferative effects on HNSCC cell lines in vitro and decreased tumorigenicity in vivo. Although, no difference in cell cycle distribution was detected between the cells transiently expressed TGM3 protein or not. This unanticipated result is most likely due to that exogenous TGM3 expression in HNSCC cell lines does not affect the cell cycle-related proteins such as cyclin D1, p53 and CDK1. We propose that the suppressive effect on tumorigenicity is possibly a result of the remarkable induction of cell apoptosis. The reduced protein level of full-length PARP, procaspase-3, procaspase- 8 and $\mathrm{Bcl}-2$ and the increased protein level of cleaved PARP and Bax in the TGM3-expressing HNSCC cell lines, as shown by western blot analysis, confirmed that the induction of apoptosis is closely involved in the effects of ectopic TGM3 expression. However, further study is required to elucidate its precise mechanism. 


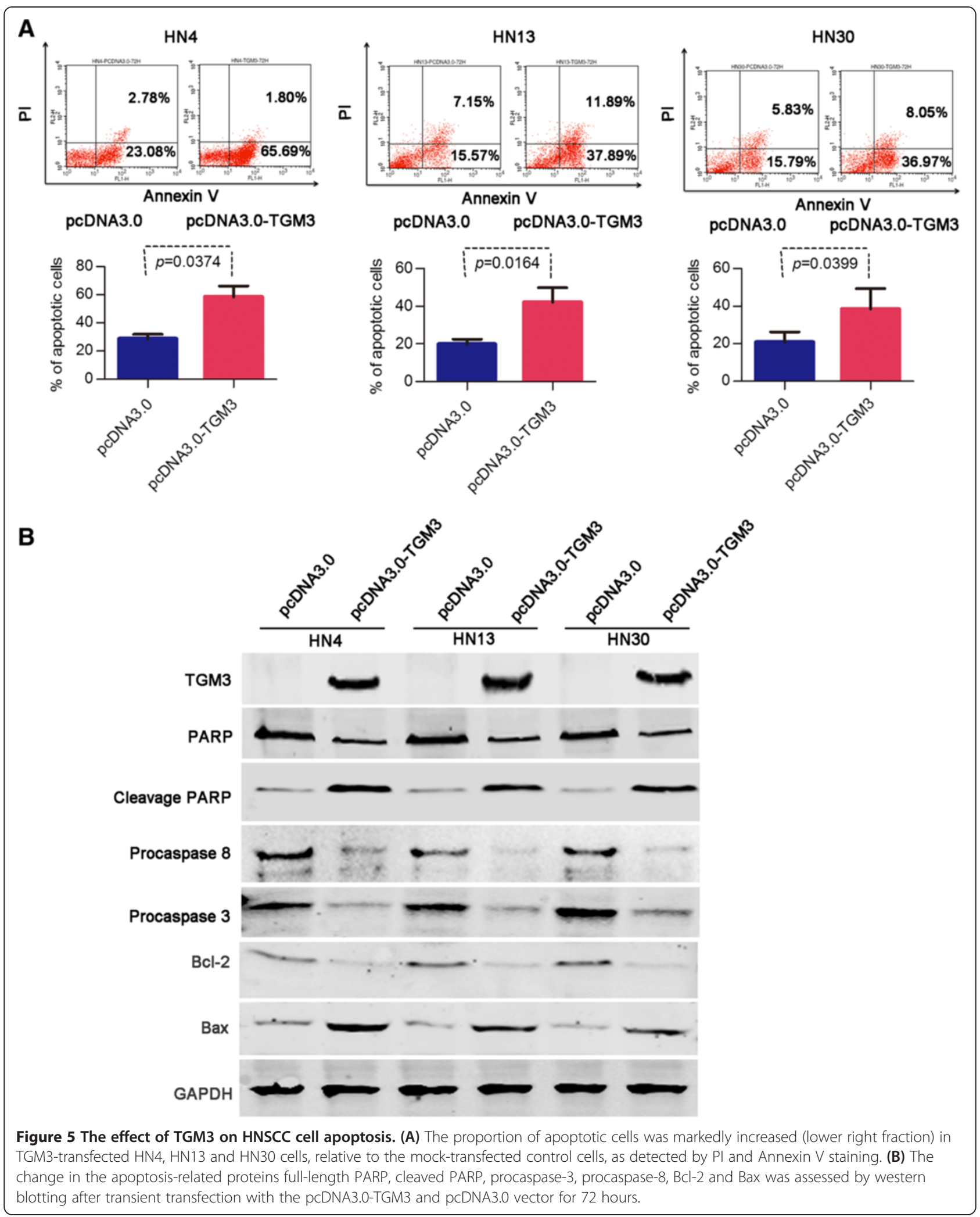




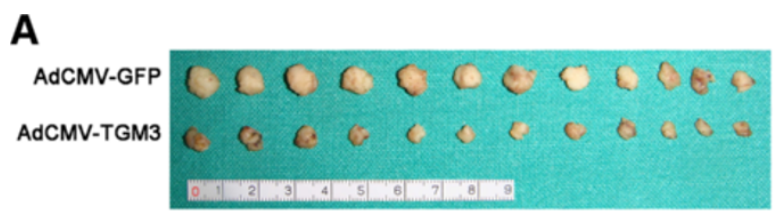

Figure 6 The effect of TGM3 on tumorigenicity in vivo. (A) Exogenous TGM3 expression inhibited xenograft tumor growth of HN30 cells treated with AdCMV-TGM3 relative to HN30 cells treated with AdCMV-GFP. Tumors' weights were measured (mean \pm SD).

\section{Conclusions}

In summary, our study has shown that the silencing of TGM3 by DNA hypermethylation is functionally involved in HNSCC cell proliferation and apoptosis. Moreover, in this study, we have shown that TGM3 expression is significantly associated with pathological differentiation of HNSCC and that TGM3 expression is an independent predictor of clinical outcome in patients with HNSCC. Our results strongly suggest that the TGM3 may be a candidate tumor suppressor and may act as a potential and novel biomarker for HNC.

\section{Materials and methods \\ Cell culture}

As previously described [44], the human head and neck squamous cell carcinoma cell lines WSU-HN4, HN6, HN12, HN13 and HN30 (kindly provided by the University of Maryland Dental School, USA) and CAL-27 (American Type Culture Collection, ATCC, USA) were cultured in Dulbecco's modified Eagle medium (DMEM; GIBCOBRL, USA) supplemented with $10 \%$ heat-inactivated FBS (GIBCO-BRL), penicillin (100 units/ml), and streptomycin $(100 \mu \mathrm{g} / \mathrm{ml})$ at $37^{\circ} \mathrm{C}$ in a humidified $5 \% \mathrm{CO} 2$ atmosphere. SCC-4, SCC-9 and SCC-25 cells (purchased from the ATCC) were cultured in DMEM/F12 medium (GIBCOBRL) supplemented with $10 \%$ heat-inactivated FBS, penicillin (100 units $/ \mathrm{ml})$, and streptomycin $(100 \mu \mathrm{g} / \mathrm{ml})$. Normal primary head and neck epithelial cells were cultured in keratinocyte serum-free medium (KSF; GIBCO-BRL) with $0.2 \mathrm{ng} / \mathrm{mL}$ recombinant epidermal growth factor (rEGF; Invitrogen, USA).

\section{Patients and specimens}

Samples were obtained from a cohort of 53 patients who were diagnosed with primary HNSCC and underwent initial surgical treatment in the Department of Oral and Maxillofacial-Head and Neck Oncology, Ninth People's Hospital, Shanghai Jiao Tong University School of Medicine (Shanghai, China) between June 2008 and December 2009. Half of each sample was embedded in paraffin for pathologic examination and immunohistochemical staining, and the other half was quickly frozen in liquid nitrogen until total RNA and protein was extracted. In addition, a tissue microarray was constructed with a donor block of paraffin-embedded tissues from 101 patients who were histologically diagnosed with primary HNSCC and underwent initial surgical treatment between 1989 and 1993. The medical records of the patients were retrospectively reviewed for inclusion and exclusion criteria. Exclusion criteria included preoperative radiotherapy and/ or chemotherapy, distant metastasis, recurrent tumors and incomplete medical records. The clinicopathologic characteristics of 101 patients are summarized in Additional file 1: Table S1. All 101 patients had clinical information and 99 patients had follow-up data. The median follow-up time was 73 months. In this study, samples from each patient were stained with hematoxylin and eosin, classified histologically, and staged according to the TNM classification system of the International Union Against Cancer (1988) prior to further analysis. The study was approved by the Institutional Ethics Committee of the Ninth People's Hospital, Shanghai Jiao Tong University School of Medicine (number: 2012-91) and all of the patients signed written informed consent in accordance with the institutional guidelines.

\section{Immunohistochemical analysis}

Immunohistochemical staining was performed using a rabbit polyclonal TGM3 antibody (Sigma, USA 1:50). The avidin-biotin complex (ABC) technique was used following the manufacturer's instructions for the Vectastatin elite ABC kit (Vector Laboratories, USA). Briefly, tissue microarrays were deparaffinized in xylene, rehydrated in graded ethanol, treated with citrate buffer for heat-induced epitope retrieval, incubated overnight at $4^{\circ} \mathrm{C}$ with rabbit polyclonal TGM3 antibody followed by incubation with a biotinylated secondary antibody and $A B C$ reagent. Twelve patients were excluded due to the samples shedding in the process of antigen retrieval and an insufficient number of tumor cells in the sections. Image-pro plus 6.0 (IPP 6.0) software was used to quantitatively determine the integrated optical density (IOD) of TGM3 staining. The procedure was performed as described in our previous study [45]. TGM3 expression was determined by randomly selecting five tumor cell areas of each specimen under 
the same conditions including light source, gain, brightness, color saturation, and contrast, then the function of irregular automated optical inspection was used by IPP 6.0 software to score. The median score of all specimen was 4683 (range 51-18083), which was set as the cut-off value to determine high or low TGM3 expression; thus, a value $>4683$ was considered high expression and a value $\leq 4683$ was considered low expression.

\section{Semiquantitative reverse transcriptase-polymerase chain reaction analysis}

Total RNA was extracted with Trizol reagent (Invitrogen) and $1 \mu \mathrm{g}$ total RNA was reverse transcribed into complementary DNA (cDNA) in a total volume of $20 \mu \mathrm{l}$ using the PrimerScript RT reagent Kit (Takara, China). One microliter of each RT reaction mixture was then subjected to PCR amplification using EX Taq DNA polymerase (Takara). The primer sequences were as follows: for TGM3, the forward primer was $5^{\prime}$-TCAACTGGCAGAC GGCCTTCA-3' and the reverse was 5'-GTACCGTCC TATGGGTGCGCT-3'; for $\beta$-actin, the forward primer was 5'-TCACCCACACTGTGCCCATCTACGA-3', and the reverse primer was 5'-CAGCGGAACCGCTCATT GCCAATGG-3'. An initial denaturation step was performed for 5 minutes at $96^{\circ} \mathrm{C}$, and 35 cycles were performed with the following PCR program: denaturing at $96^{\circ} \mathrm{C}$ for 15 seconds, annealing at $56^{\circ} \mathrm{C}$ for 30 seconds, and elongating at $72^{\circ} \mathrm{C}$ for 50 seconds. The program was completed with a final extension step at $72^{\circ} \mathrm{C}$ for 5 minutes.

\section{Real-time polymerase chain reaction analysis}

All real-time PCR reactions were performed using an ABI 7300 real-time PCR system (Applied Biosystems, USA) and the SYBR Premix Ex Taq reagent kit (Takara). The primer sequences were as follows: for TGM3, the forward primer was 5'-GACAAGTTCTCCAGCCAG GAG-3', and the reverse primer was 5'-AGTGGAAA CACAGCCTTCGTC-3'; for $\beta$-actin, the forward primer was 5'-CCTGGCACCCAGCACAAT-3', and the reverse primer was 5'-GGGCCGGACTCGTCATACT-3'. Realtime PCR was performed in a final volume of $20 \mu \mathrm{l}$ with $1 \mu \mathrm{l}$ of template cDNA at a concentration of $20 \mathrm{ng} / \mu \mathrm{l}$ with $10 \mu \mathrm{l} \mathrm{SYBR}$ Premix Ex Taq, $0.4 \mu \mathrm{l}$ ROX Reference Dye and $0.2 \mu \mathrm{M}$ of each primer for the target gene and the internal control ( $\beta$-actin). Thermal cycling conditions were $95^{\circ} \mathrm{C}$ for 30 seconds followed by 40 cycles of $95^{\circ} \mathrm{C}$ for 5 seconds and $60^{\circ} \mathrm{C}$ for 31 seconds. The results of real-time PCR were defined as $\mathrm{Ct}$ values, which represent the cycle number at which the fluorescent signal of the samples passes a given threshold above the baseline. $\Delta \mathrm{Ct}$ was the difference in the $\mathrm{Ct}$ values derived from the specific genes compared with $\beta$-actin of the same sample. The relative expression level was determined as $2^{-\Delta \Delta \mathrm{Ct}}$, where $\Delta \Delta \mathrm{Ct}=\Delta \mathrm{Ct}$ (HNSCC samples) $-\Delta \mathrm{Ct}$ (adjacent normal sample), thereby indicating the fold change in the HNSCC samples relative to adjacent normal samples. The significance level was set at $P<0.05$.

\section{Induction of gene expression by 5-Aza-dC and TSA}

As previously described [46], to induce TGM3 expression, the above-mentioned HNSCC cell lines were treated with $2 \mu \mathrm{M}$ or $5 \mu \mathrm{M} 5$ '-aza-2'-deoxycytidine (5-Aza-dC), a DNA demethylation reagent, for 120 hours and $0.5 \mu \mathrm{M}$ trichostatin A (TSA), a specific inhibitor of histone deacetylase for 48 hours.

\section{Cytosine methylation analysis}

MethPrimer online software (http://www.urogene.org/ index.html) was used to discover the $\mathrm{CpG}$ islands of the TGM3 promoter and to design bisulfate-sequencing PCR (BSP) primers. Genomic DNAs of HNSCC samples and cell lines were treated with the MethylCodeTM Bisulfite Conversion Kit (Invitrogen) and the converted DNA was subjected to PCR using BSP primers (forward, 5'-GTTTAGGTTGGAGTGTAGTGATG-3'; reverse, 5' TCACTTAAAACCAAAAATTCAAAAC-3'). The PCR products were visualized by $2 \%$ agarose gel electrophoresis and subcloned into a pMD18-T vector (Takara). Ten colonies were sequenced using an ABI 3730 sequencer.

\section{Plasmid construction}

To construct the TGM3 expression vector, the open reading frame (ORF) of human TGM3 cDNA was cloned into the eukaryotic expression vector pcDNA3.0 (Invitrogen). The forward primer used for the amplification of the ORF of the TGM3 cDNA was 5' -TTAAAGCTTCT GAGAAGAGGCAGAGGAAG-3, containing a HindIII site; the reverse primer was 5'-TATGAATTCTGTACGG GAGGCCACCAGCGC- 3, containing an EcoRI site without the stop codon. The amplified product of the TGM3 gene was purified, digested and ligated into the respective HindIII and EcoRI sites in the pcDNA3.0 vector.

\section{Adenoviral vectors}

AdCMV-TGM3, a recombinant adenovirus expressing the TGM3, was constructed in a replication-deficient E1 and E3 adenovirus vector. Briefly, the complete coding sequence of the TGM3 gene was subcloned into the AdCMV vector. Then, the recombinant clones of shuttle plasmid and viral-backbone plasmid were packaged in HEK293 cells. The viral particles were purified by cesium chloride density gradient centrifugation and titered using the End-Point Dilution Assay. AdCMV-GFP, which only expresses GFP, was used as the control adenovirus.

\section{Western blot analysis}

Cells were harvested in SDS lysis buffer (Beyotime, China), and cell lysates were electrophoresed through 
$10 \%-15 \%$ polyacrylamide gels and transferred to a nitrocellulose membrane. The membrane was incubated with a rabbit polyclonal TGM3 antibody (Sigma, USA 1:250), a rabbit polyclonal poly (ADP-ribose) polymerase (PARP) antibody, which recognize the $116-\mathrm{kDa}$ full-length PARP protein (Abcam, USA 1:200), a rabbit monoclonal PARP antibody, which recognizes the $85-\mathrm{kDa}$ cleaved PARP protein (Epitomics, USA 1:1000), a rabbit polyclonal caspase-8 antibody, which recognizes the $55-\mathrm{kDa}$ procaspase- 8 protein (Abcam, USA 1:1000), a rabbit polyclonal caspase-3 antibody, which recognizes the $32-\mathrm{kDa}$ procaspase-3 protein (Abcam, USA 1:500), a rabbit monoclonal Bcl-2 antibody (Epitomics, USA 1:500) and a rabbit monoclonal Bax antibody (Epitomics, USA 1:500). A mouse monoclonal GAPDH antibody (Sigma, USA, 1:10,000) was used throughout as a loading control. The secondary antibodies were labeled with IRDye ${ }^{\mathrm{Tm}} 800$, and signals were observed using an Odyssey Infrared Imaging System (Rockland, USA).

\section{Cell proliferation assay}

Cell-proliferation assays were performed to analyze the proliferation ability of WSU-HN4, HN13 and HN30 cells transiently transfected with the pcDNA3.0-TGM3 vector or the pcDNA3.0 empty vector. Briefly, the cells were plated in 96-well plates at $3 \times 10^{3}$ cells per well and maintained at $37^{\circ} \mathrm{C}$ in a humidified incubator. Ten microliters of Cell-Counting Kit (CCK)-8 (Dojindo, Japan) solution was added at 24, 48, 72, 96 and 120 hours into triplicate wells and incubated for 1.5 hours. The number of viable cells in each well was measured by reading the optical density (OD) of absorbance at $450 \mathrm{~nm}$. Measurements were performed in triplicate.

\section{Colony formation assay}

Twenty-four hours after transiently transfecting with the pcDNA3.0-TGM3 vector or the pcDNA3.0 empty vector, $1 \times 10^{3}$ cells were plated in $60-\mathrm{mm}$ culture dishes and incubated with $500 \mu \mathrm{g} / \mathrm{ml} \mathrm{G} 418$ for 20 days to allow for colony formation. The colonies were fixed with $70 \%$ ethanol for 1 hour, stained with $0.1 \%$ Coomassie Brilliant Blue R-250 (Thermo, USA) for 2 hours and washed with PBS. Colonies of more than 50 cells were counted under a dissecting microscope. The data from the colony formation assays were calculated as the means $( \pm S D)$ from 3 independent experiments, each performed in triplicate.

\section{Apoptosis assay}

The cells transiently transfected with the pcDNA3.0TGM3 vector or pcDNA3.0 empty vector were harvested at 24, 48 and 72 hours. These cells were then quantified by flow cytometry using the FITC Annexin V Apoptosis Detection Kit (BD Biosciences, USA) according to the manufacturer's protocols. Briefly, trypsinized adherent cells and floating cells were harvested, washed twice with cold PBS and resuspended in $1 \times$ Binding Buffer at a concentration of $1 \times 10^{6}$ cells $/ \mathrm{ml}$. Then, $5 \mu \mathrm{l}$ of FITC Annexin $\mathrm{V}$ and $5 \mu \mathrm{l}$ propidium iodide (PI) were added, and the cells were incubated for 15 minutes at $25^{\circ} \mathrm{C}$ in the dark. The cells were then resuspended in $400 \mu \mathrm{l}$ of $1 \times$ Binding Buffer and analyzed immediately by BD LSR II flow cytometry (BD Biosciences, USA).

\section{Tumorigenicity assay in vivo}

To evaluate the anti-tumor effect of TGM3 in vivo, a HNSCC xenograft model was generated in BALB/C nude mice ( 4 weeks old) by subcutaneously injecting $4 \times 10^{6}$ HN30 cells into the left and right axilla. One week after the injections, all mice were divided randomly into 2 groups (6 mice per group) and were treated with a multiple-center intratumor injection of $1 \times 10^{9} \mathrm{Pfu}$ (plaque forming units) of AdCMV-TGM3 or AdCMV-GFP 2 times a week for up to 2 weeks. One week after the treatments, the animals were sacrificed, and the tumor volume and tumor weight were measured. Differences in tumor weights were analyzed using Student's $t$-test.

\section{Statistical analysis}

For immunohistochemical analysis, the Student $t$-test and one-way ANOVA were used to analyze the associations between TGM3 expression levels and patient characteristics. The log-rank test was used to analyze univariate associations between the expression levels of TGM3 and overall survival. The Cox proportional hazards model was used for multivariate analyses, and all potential prognostic factors with $P$ values $<0.05$ from the univariate analysis were incorporated into multivariate analyses. The results of the real-time PCR, cell proliferation assay, colony formation assay and apoptosis assay were evaluated using Student's $t$-tests. All tests were two-sided, and $P$ values less than 0.05 were considered statistically significant.

\section{Additional file}

Additional file 1: Table S1. Clinicopathologic characteristics of 101 patients.

\section{Abbreviations}

TGM3: Transglutaminase 3; HNC: Head and neck cancer; HNSCC: Head and neck squamous cell carcinoma; OSCC: Oral squamous cell carcinoma; ESCC: Esophageal squamous cell carcinoma; TSS: Transcriptional start site; TGMs: Transglutaminases; Cl: Confidence interval; HR: Hazard ratio; pN: Pathological lymph node status; TNM: Tumor-lymph node-metastasis classification; ATCC: American Type Culture Collection; ABC: Avidin-biotin complex; IOD: Integrated optical density; 5-Aza-dC: 5'-aza-2'-deoxycytidine; TSA: Trichostatin A; BSP: Bisulfate-sequencing PCR; ORF: Open reading frame; Pl: Propidium iodide.

\section{Competing interests}

The authors declare that they have no competing interests. 


\section{Authors' contributions}

XBW, WC, XW, JJZ, QX, ZJL and WTC performed experiments; WTC, YDW and JJZ participated in the design of the study; XBW, XW and WC participated in data analysis and statistical analysis; XBW, YDW and WTC drafted and revised the manuscript. All authors read and approved the final manuscript

\section{Acknowledgements}

This study was supported by the National Natural Science Foundation of China (30973343, 81072171 and 91229103) and by projects of the Shanghai Science and Technology Committee (11DZ2291800, 10DZ1951300 and 10XD1402500)

Received: 2 August 2013 Accepted: 25 November 2013

Published: 1 December 2013

\section{References}

1. Mignogna MD, Fedele S, Lo Russo L: The World Cancer Report and the burden of oral cancer. Eur J Cancer Prev 2004, 13:139-142.

2. Forastiere A, Koch W, Trotti A, Sidransky D: Head and neck cancer. N Engl J Med 2001, 345:1890-1900.

3. Le Tourneau C, Faivre S, Siu LL: Molecular targeted therapy of head and neck cancer: review and clinical development challenges. Eur J Cancer 2007, 43:2457-2466.

4. Wheeler SE, Suzuki S, Thomas SM, Sen M, Leeman-Neill RJ, Chiosea SI, Kuan CT, Bigner DD, Gooding WE, Lai SY, Grandis JR: Epidermal growth factor receptor variant III mediates head and neck cancer cell invasion via STAT3 activation. Oncogene 2010, 29:5135-5145.

5. Chung CH, Parker JS, Karaca G, Wu J, Funkhouser WK, Moore D, Butterfoss D, Xiang D, Zanation A, Yin X, et al: Molecular classification of head and neck squamous cell carcinomas using patterns of gene expression. Cancer Cell 2004, 5:489-500.

6. Bentzen SM, Atasoy BM, Daley FM, Dische S, Richman PI, Saunders MI, Trott KR, Wilson GD: Epidermal growth factor receptor expression in pretreatment biopsies from head and neck squamous cell carcinoma as a predictive factor for a benefit from accelerated radiation therapy in a randomized controlled trial. J Clin Oncol 2005, 23:5560-5567.

7. Hanahan D, Weinberg RA: Hallmarks of cancer: the next generation. Cell 2011, 144:646-674.

8. Mohr S, Leikauf GD, Keith G, Rihn BH: Microarrays as cancer keys: an array of possibilities. J Clin Oncol 2002, 20:3165-3175.

9. Belbin TJ, Singh B, Smith RV, Socci ND, Wreesmann VB, Sanchez-Carbayo M, Masterson J, Patel S, Cordon-Cardo C, Prystowsky MB, Childs G: Molecular profiling of tumor progression in head and neck cancer. Arch Otolaryngol Head Neck Surg 2005, 131:10-18.

10. Chin D, Boyle GM, Williams RM, Ferguson K, Pandeya N, Pedley J, Campbell CM, Theile DR, Parsons PG, Coman WB: Novel markers for poor prognosis in head and neck cancer. Int J Cancer 2005, 113:789-797.

11. Kuriakose MA, Chen WT, He ZM, Sikora AG, Zhang P, Zhang ZY, Qiu WL, Hsu DF, McMunn-Coffran C, Brown SM, et al: Selection and validation of differentially expressed genes in head and neck cancer. Cell Mol Life Sci 2004, 61:1372-1383.

12. Hitomi K, Horio Y, Ikura K, Yamanishi K, Maki M: Analysis of epidermal-type transglutaminase (TGase 3) expression in mouse tissues and cell lines. Int J Biochem Cell Biol 2001, 33:491-498.

13. Hitomi K: Transglutaminases in skin epidermis. Eur J Dermatol 2005, 15:313-319.

14. Hitomi K, Presland RB, Nakayama T, Fleckman P, Dale BA, Maki M: Analysis of epidermal-type transglutaminase (transglutaminase 3 ) in human stratified epithelia and cultured keratinocytes using monoclonal antibodies. J Dermatol Sci 2003, 32:95-103.

15. Kalinin AE, Kajava AV, Steinert PM: Epithelial barrier function: assembly and structural features of the cornified cell envelope. Bioessays 2002 24:789-800

16. Eckert RL, Sturniolo MT, Broome AM, Ruse M, Rorke EA: Transglutaminase function in epidermis. J Invest Dermatol 2005, 124:481-492.

17. He G, Zhao Z, Fu W, Sun X, Xu Z, Sun K: Study on the loss of heterozygosity and expression of transglutaminase 3 gene in laryngeal carcinoma. Zhonghua Yi Xue Yi Chuan Xue Za Zhi 2002, 19:120-123.

18. Uemura N, Nakanishi Y, Kato H, Saito S, Nagino M, Hirohashi S, Kondo T: Transglutaminase 3 as a prognostic biomarker in esophageal cancer revealed by proteomics. Int J Cancer 2009, 124:2106-2115.
19. Negishi A, Masuda M, Ono M, Honda K, Shitashige M, Satow R, Sakuma T, Kuwabara H, Nakanishi Y, Kanai Y, et al: Quantitative proteomics using formalin-fixed paraffin-embedded tissues of oral squamous cell carcinoma. Cancer Sci 2009, 100:1605-1611.

20. Mendez E, Fan W, Choi P, Agoff SN, Whipple M, Farwell DG, Futran ND, Weymuller EA Jr, Zhao LP, Chen C: Tumor-specific genetic expression profile of metastatic oral squamous cell carcinoma. Head Neck 2007, 29:803-814.

21. Lu SL, Herrington H, Reh D, Weber S, Bornstein S, Wang D, Li AG, Tang CF, Siddiqui $Y$, Nord J, et al: Loss of transforming growth factor-beta type II receptor promotes metastatic head-and-neck squamous cell carcinoma. Genes Dev 2006, 20:1331-1342.

22. Hasina R, Hulett K, Bicciato S, Di Bello C, Petruzzelli GJ, Lingen MW: Plasminogen activator inhibitor-2: a molecular biomarker for head and neck cancer progression. Cancer Res 2003, 63:555-559.

23. Erjala K, Sundvall M, Junttila T, Zhang N, Savisalo M, Mali P, Kulmala J, Pulkkinen J, Grenman R, Elenius K: Signaling via ErbB2 and ErbB3 associates with resistance and epidermal growth factor receptor (EGFR) amplification with sensitivity to EGFR inhibitor gefitinib in head and neck squamous cell carcinoma cells. Clin Cancer Res 2006, 12:4103-4111.

24. Van Tubergen E, Vander Broek R, Lee J, Wolf G, Carey T, Bradford C, Prince M, Kirkwood KL, D'Silva NJ: Tristetraprolin regulates interleukin-6, which is correlated with tumor progression in patients with head and neck squamous cell carcinoma. Cancer 2011, 117:2677-2689.

25. Han J, Kioi M, Chu WS, Kasperbauer JL, Strome SE, Puri RK: Identification of potential therapeutic targets in human head \& neck squamous cell carcinoma. Head Neck Oncol 2009, 1:27.

26. Grandis JR: Prognostic biomarkers in head and neck cancer. Clin Cancer Res 2006, 12:5005-5006.

27. Schaaij-Visser TB, Graveland AP, Gauci S, Braakhuis BJ, Buijze M, Heck AJ, Kuik DJ, Bloemena E, Leemans CR, Slijper M, Brakenhoff RH: Differential proteomics identifies protein biomarkers that predict local relapse of head and neck squamous cell carcinomas. Clin Cancer Res 2009, $15: 7666-7675$

28. Molling JW, Langius JA, Langendijk JA, Leemans $C R$, Bontkes $H J$, van der Vliet HJ, von Blomberg BM, Scheper RJ, van den Eertwegh AJ: Low levels of circulating invariant natural killer T cells predict poor clinical outcome in patients with head and neck squamous cell carcinoma. J Clin Oncol 2007, 25:862-868

29. Petrik D, Lavori PW, Cao H, Zhu Y, Wong P, Christofferson E, Kaplan MJ, Pinto HA, Sutphin P, Koong AC, et al: Plasma osteopontin is an independent prognostic marker for head and neck cancers. $J$ Clin Oncol 2006, 24:5291-5297.

30. Marsit CJ, Posner MR, McClean MD, Kelsey KT: Hypermethylation of E-cadherin is an independent predictor of improved survival in head and neck squamous cell carcinoma. Cancer 2008, 113:1566-1571.

31. Liu J, Zhou Y, Wan J, Liu Z: Expression of TGM3 protein and its significance in laryngeal carcinoma. Lin Chung Er Bi Yan Hou Tou Jing Wai Ke Za Zhi 2012, 26:101-103.

32. Lallemant B, Evrard A, Combescure C, Chapuis H, Chambon G, Raynal C, Reynaud C, Sabra O, Joubert D, Hollande F, et al: Clinical relevance of nine transcriptional molecular markers for the diagnosis of head and neck squamous cell carcinoma in tissue and saliva rinse. BMC Cancer 2009, 9:370.

33. Ye H, Yu T, Temam S, Ziober BL, Wang J, Schwartz JL, Mao L, Wong DT, Zhou X: Transcriptomic dissection of tongue squamous cell carcinoma. BMC Genomics 2008, 9:69.

34. Chen BS, Wang MR, Xu X, Cai Y, Xu ZX, Han YL, Wu M: Transglutaminase-3, an esophageal cancer-related gene. Int J Cancer 2000, 88:862-865.

35. Gonzalez HE, Gujrati M, Frederick M, Henderson Y, Arumugam J, Spring PW, Mitsudo K, Kim HW, Clayman GL: Identification of 9 genes differentially expressed in head and neck squamous cell carcinoma. Arch Otolaryngol Head Neck Surg 2003, 129:754-759.

36. Luo A, Kong J, Hu G, Liew CC, Xiong M, Wang X, Ji J, Wang T, Zhi H, Wu M, Liu Z: Discovery of Ca2 + -relevant and differentiation-associated genes downregulated in esophageal squamous cell carcinoma using CDNA microarray. Oncogene 2004, 23:1291-1299.

37. Ohkura S, Kondoh N, Hada A, Arai M, Yamazaki Y, Sindoh M, Takahashi M, Matsumoto I, Yamamoto M: Differential expression of the keratin-4, -13, -14, -17 and transglutaminase 3 genes during the development of oral squamous cell carcinoma from leukoplakia. Oral Oncol 2005, 41:607-613. 
38. Liu W, Yu ZC, Cao WF, Ding F, Liu ZH: Functional studies of a novel oncogene TGM3 in human esophageal squamous cell carcinoma. World J Gastroenterol 2006, 12:3929-3932.

39. Kondoh N, Ishikawa T, Ohkura S, Arai M, Hada A, Yamazaki Y, Kitagawa Y, Shindoh M, Takahashi M, Ando T, et al: Gene expression signatures that classify the mode of invasion of primary oral squamous cell carcinomas. Mol Carcinog 2008, 47:744-756.

40. Choi P, Jordan CD, Mendez E, Houck J, Yueh B, Farwell DG, Futran N, Chen C: Examination of oral cancer biomarkers by tissue microarray analysis. Arch Otolaryngol Head Neck Surg 2008, 134:539-546.

41. Lorand L, Graham RM: Transglutaminases: crosslinking enzymes with pleiotropic functions. Nat Rev Mol Cell Biol 2003, 4:140-156.

42. Griffin M, Casadio R, Bergamini CM: Transglutaminases: nature's biological glues. Biochem J 2002, 368:377-396.

43. Candi E, Schmidt R, Melino G: The cornified envelope: a model of cell death in the skin. Nat Rev Mol Cell Biol 2005, 6:328-340.

44. Cao W, Feng Z, Cui Z, Zhang C, Sun Z, Mao L, Chen W: Up-regulation of enhancer of zeste homolog 2 is associated positively with cyclin D1 overexpression and poor clinical outcome in head and neck squamous cell carcinoma. Cancer 2012, 118:2858-2871.

45. Feng Z, Guo W, Zhang C, Xu Q, Zhang P, Sun J, Zhu H, Wang Z, Li J, Wang L, et al: CCND1 as a predictive biomarker of neoadjuvant chemotherapy in patients with locally advanced head and neck squamous cell carcinoma. PLoS One 2011, 6:e26399.

46. Cao W, Zhang ZY, Xu Q, Sun Q, Yan M, Zhang J, Zhang P, Han ZG, Chen WT: Epigenetic silencing of MAL, a putative tumor suppressor gene, can contribute to human epithelium cell carcinoma. Mol Cancer 2010, 9:296.

doi:10.1186/1476-4598-12-151

Cite this article as: Wu et al:: TGM3, a candidate tumor suppressor gene, contributes to human head and neck cancer. Molecular Cancer 2013 12:151.

\section{Submit your next manuscript to BioMed Central and take full advantage of:}

- Convenient online submission

- Thorough peer review

- No space constraints or color figure charges

- Immediate publication on acceptance

- Inclusion in PubMed, CAS, Scopus and Google Scholar

- Research which is freely available for redistribution 